\title{
Thin Ge buffer layer on Silicon for Integration of III-V on Silicon
}

Junjie Yang ${ }^{1}$, Pamela Jurczak ${ }^{1 *}$, Fan Cui ${ }^{1}$, Keshuang $\mathrm{Li}^{1}$, Mingchu Tang ${ }^{1}$, Luke Billiald ${ }^{2}$, Richard Beanland ${ }^{2}$, Ana M. Sanchez ${ }^{2}$, and Huiyun Liu ${ }^{1}$

${ }^{1}$ Department of Electronic and Electrical Engineering, University College London, Torrington Place, WC1E 7JE London, United Kingdom

${ }^{2}$ Department of Physics, University of Warwick, CV4 7AL, Coventry, United Kingdom

Abstract - Development of Si-based lasers is considered as the key to the realization of fully integrated Si photonic circuits. Monolithic growth of III-V lasers on Si substrates is one of the most promising solutions for developing a commercially viable Si-based laser. However, the performances of current devices are still hindered by defects, hence the optimisation of crystal quality of the laser structures is of paramount importance. This paper reports on growth optimisation of thin Ge buffer layers on $\mathrm{Si}$ as an alternative to thick GaAs buffer layers. This method reduces the overall thickness and lowers the threading dislocation density in III-V semiconductors integrated on silicon platform.

Keywords-III-V-Silicon integration, Ge buffer layers, Si photonics

\section{Introduction}

Silicon-based photonic integrated circuits promise low-cost, low-power and high-bandwidth solutions for high-speed interconnects and optical communication systems thanks to the maturity of CMOS processing technology $[1,2]$. However, the lack of a reliable silicon based light source prevents full optical integration hence realizing a silicon laser has been considered the "Holy Grail" for silicon photonics [2-5]. Due to its indirect bandgap property, bulk silicon material is very inefficient at emitting light. In contrast, III-V semiconductors with direct bandgaps have superior light emission efficiencies [6-8]. The epitaxial growth of III-Vs on Si substrates has been regarded as one of the best ways of realizing a high-performance light source for Si-based photonic integrated circuits. Such a solution faces several challenges due to large material dissimilarities between the III-V and group-IV semiconductors. The large lattice mismatch, different polarities and thermal expansion coefficients between impede efficient operation of the devices by introducing threading dislocations (TDs), anti-phase boundaries (APBs) and thermal cracks [9-12].

Various techniques, including growing thick III-V buffer layers with dislocation filter layers (DFLs) have been used to improve the material quality of the III-V photonic devices on Si [13-15]. Implementing quantum confined materials, such as quantum wells and quantum dots (QDs) [16-19], has allowed for further improvements in the devices quality and realization of the first functional lasers [20]. Recently, a long lifetime electrically-pumped continuous-wave III-V quantum dot laser monolithically grown on silicon has been successfully demonstrated by Chen et al [7]. Such a laser can be successfully integrated with the silicon photonic components to create high-performance wafer-scale fully photonic circuits. The use of thick GaAs buffer layers, however, has several drawbacks. Firstly, the thick III-V layers can lead to crack for releasing the built-up thermal strain. Furthermore, the thick buffer layer increases both the cost and growth time of such devices, as well as introducing difficulties for optical coupling to a waveguide, making integration of III-V lasers on SOI more challenging. In this paper, we propose to replace the thick GaAs buffer layers with 


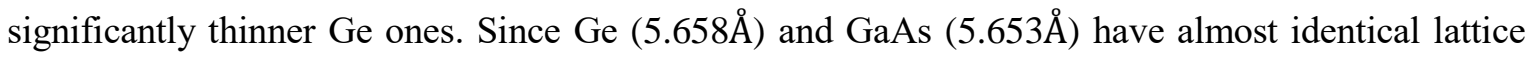
constants, no significant strain is introduced when a GaAs-based III-V laser structure is grown on Ge, compared with GaAs directly grown Si substrate [8]. Combing thin Ge buffer layer with superior light emitting properties of III-V QD laser structures could enable next generation of high-performance CMOS-compatible light sources, suitable for photonic integrated circuits.

\section{Methods and results}

Simple two-step Ge buffer layers were grown on N-type (100) silicon substrates using a solid-source molecular beam epitaxy (MBE) system. The crystallographic properties of the grown samples were studied by X-ray diffraction (XRD), electron channeling contrast imaging (ECCI) [21], and transmission electron microscopy (TEM). The surface morphology was analysed with atomic force microscopy (AFM). Due to a relatively large (4.18\%) lattice mismatch between the Ge and Si lattices, the formation of dislocations is inevitable. Minimizing the threading dislocation density (TDD) whilst obtaining smooth surfaces has been the priority for this study. The impact of annealing temperatures and the use of dopants on the quality of the Ge buffer layers have been investigated. The correlation between the thickness of the buffer layer and the TDD have also been analysed.

\subsection{Effects of annealing}

The first part of the study focused on optimizing annealing temperatures for highest quality buffer layers. A total of four $270 \mathrm{~nm}$-thick samples have been grown, as shown in Fig. 1. First, a lowtemperature (LT) layer of $30 \mathrm{~nm}$ of Ge was grown at substrate temperature of $250^{\circ} \mathrm{C}$. Then, the substrate temperature was increased to $500^{\circ} \mathrm{C}$ and a further $180 \mathrm{~nm}$ of high-temperature (HT) Ge has been grown, followed by an in-situ 30-minute annealing step. Finally, the substrate temperature was reduced to $600^{\circ} \mathrm{C}$ and a 60 -nm-thick Ge cap was grown. The four samples have been annealed at temperatures of $750,800,850$ and $900^{\circ} \mathrm{C}$ respectively.

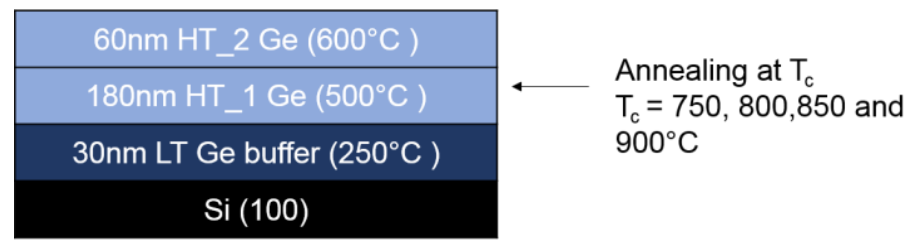

Fig. 1. Schematic structure of Ge buffer layers.

The ECCI and TEM results for those samples are shown in Fig. 2. A reduction in TDD has been observed for samples annealed at temperatures of 850 and $900^{\circ} \mathrm{C}$. A high density of misfit dislocations can be observed at the Ge/Si interface, many of them propagating further as TDs and some of them penetrating the whole structure. Due to the low total thickness of the buffer layers $(270 \mathrm{~nm})$, the dislocations have limited space in which to annihilate before they reach the surface. 

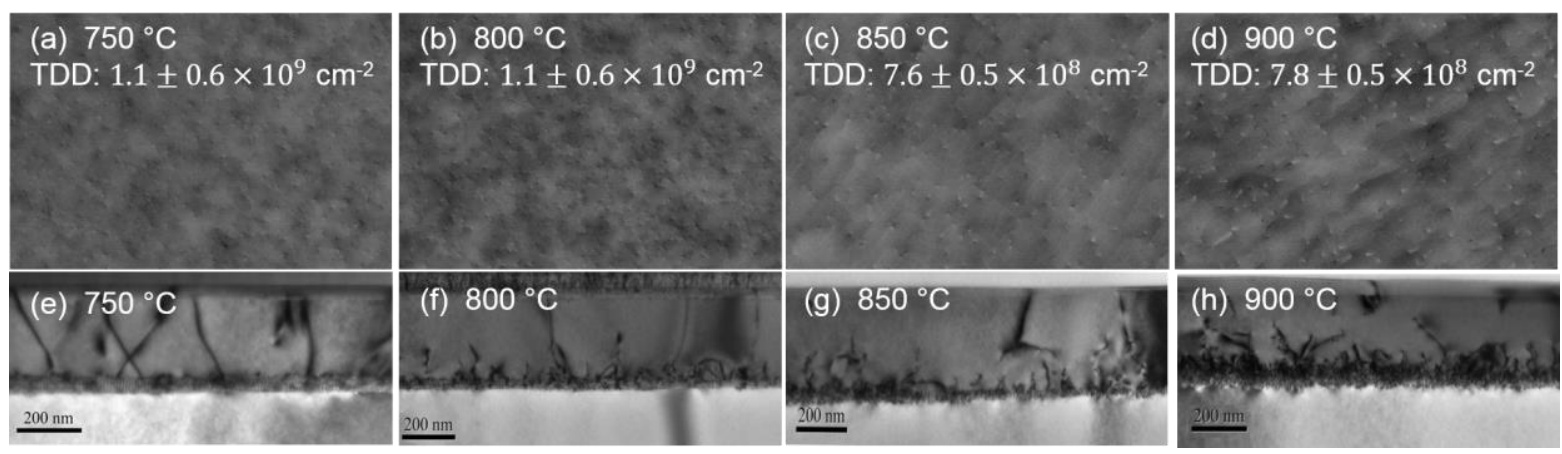

Fig. 2. Upper: ECCI and lower: TEM micrographs of Ge buffer layers annealed continuously for 30 minutes at (a) 750 , (b) 800 , (c) 850 and (d) $900^{\circ} \mathrm{C}$, showing the threading dislocations on the surface (a-d) and across the sample thickness (e-h).

Surface analysis of the samples (Fig. 3) shows that the root-mean-square (RMS) roughness of the buffer layers increases slightly with increasing annealing temperatures. However, the surface of the sample annealed at $900^{\circ} \mathrm{C}$ is significantly rougher than that of the other samples as the temperature approaches melting point of $\mathrm{Ge}$ and 3D structures are formed. Considering both TDD and RMS toughness of samples annealed at different temperatures, as shown in Fig. 4 , it suggests that $850^{\circ} \mathrm{C}$ is the optimal annealing temperature to lower the TDD whilst retaining a smooth surface.
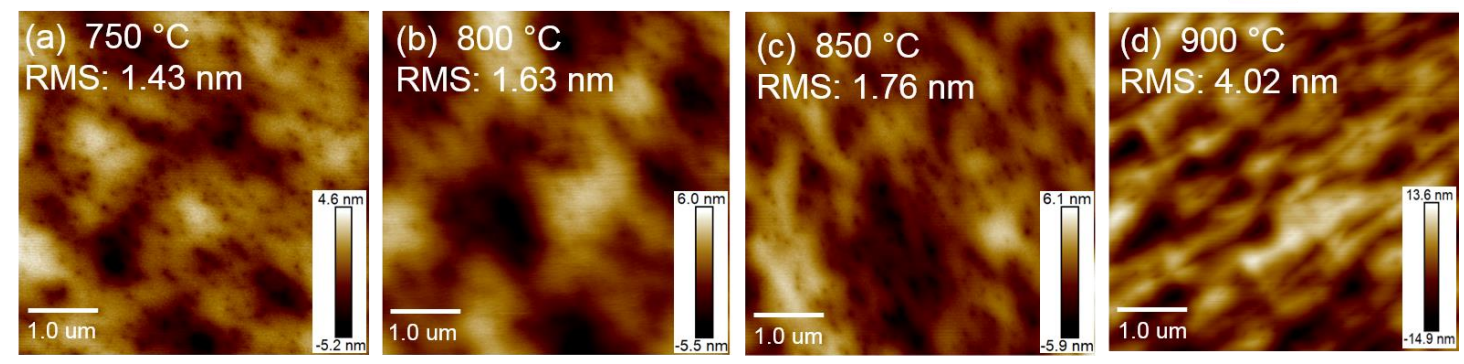

Fig. 3. $5 \mu \mathrm{m} \times 5 \mu \mathrm{m}$ AFM images of the surfaces of the buffer layers (270nm) annealed continuously for 30 minutes at $750,800,850$ and $900^{\circ} \mathrm{C}$.

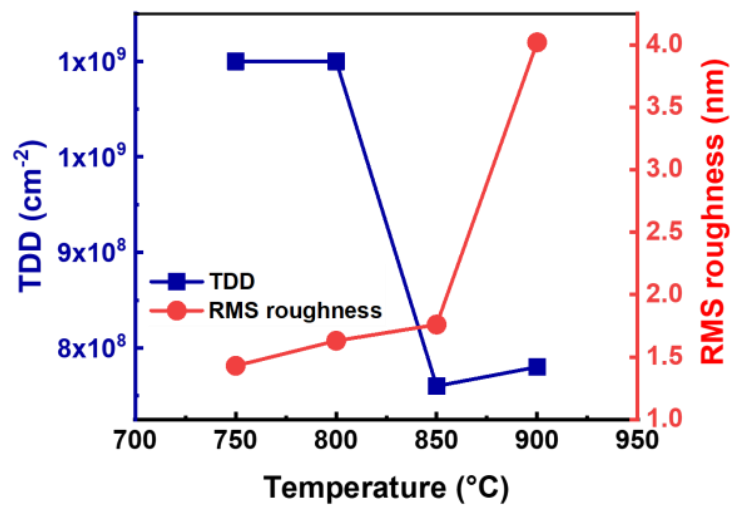

Fig. 4. Summary of TDD and RMS roughness for buffer layers (270nm) annealed continuously for 30 minutes at $750,800,850$ and $900^{\circ} \mathrm{C}$.

The composition of the samples was analyzed using an XRD method. The black line in Fig. 5 represents XRD measurement of the sample annealed at $750^{\circ} \mathrm{C}$ where two peaks can be clearly distinguished, the one on the left related to the Ge buffer layer, and the one on the right to the Si substrate. A third peak becomes visible just to the right of the Ge one when the annealing temperature is increased from 750 to $900^{\circ} \mathrm{C}$. This peak can be attributed to GeSi alloy formed at the interface between $\mathrm{Ge}$ and $\mathrm{Si}$ due to $\mathrm{Si}$ 
diffusion into Ge at high temperature. As can be seen from the green line, the third peak becomes comparable in strength to the Ge one after annealing at $900^{\circ} \mathrm{C}$, which suggests significant diffusion of $\mathrm{Si}$ deep into the Ge buffer layer. Composition analysis of the sample estimates around $10 \% \mathrm{Si}$ content in the GeSi layer.

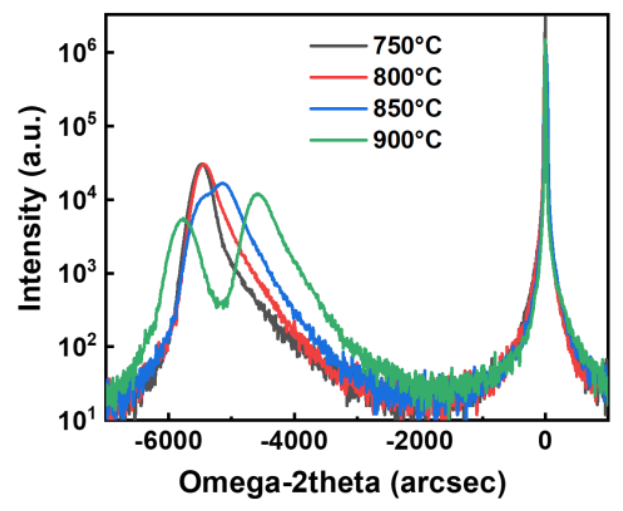

Fig. 5. XRD results for samples annealed at $750,800,850$ and $900^{\circ} \mathrm{C}$.

The next part of the annealing study focused on the impact of replacing the continuous 30 min annealing cycle with a periodic one consisting of five 5-minute anneals between high temperature $\left(\mathrm{T}_{\mathrm{H}}\right)$ of $850^{\circ} \mathrm{C}$ and low temperature $\left(\mathrm{T}_{\mathrm{L}}\right)$ of $450^{\circ} \mathrm{C}$. The TEM results show a further small reduction in TDD which attributes to the TD glide and annihilation caused by thermal stress when cooling down and heating up $[22,23]$. The AFM images also show a slight improvement in the surface smoothness of the sample. Hence all the following samples in this study have been annealed using a $5 \times 5$ minute cycle between $\mathrm{T}_{\mathrm{H}}=850^{\circ} \mathrm{C}$ and $\mathrm{T}_{\mathrm{L}}=450^{\circ} \mathrm{C}$.
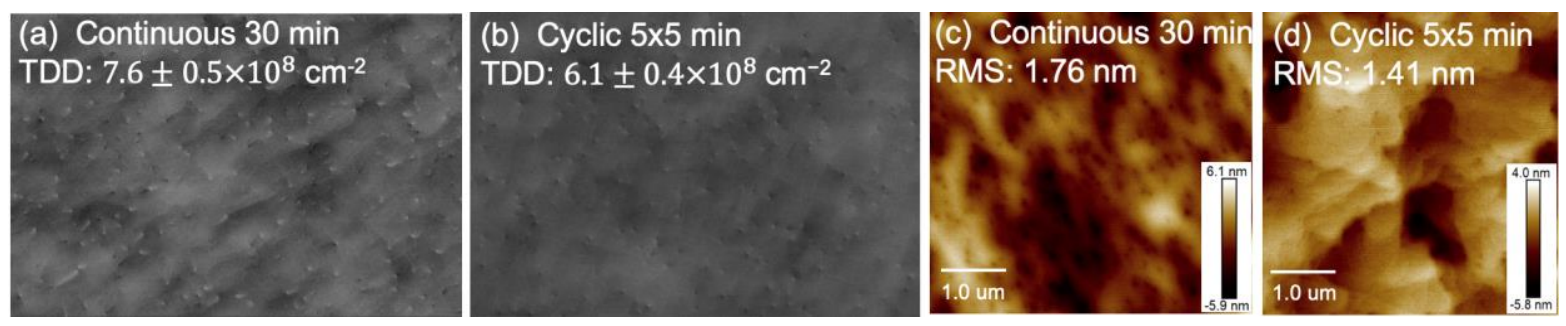

Fig. 6. A comparison between the continuous and cyclic annealing methods with ECCI showing threading dislocations and AFM images $(5 \mu \mathrm{m} \times 5 \mu \mathrm{m})$ showing surface roughness.

\subsection{Effects of thickness}

The second part of the study investigated the correlation between the thickness of the Ge buffer layers and TDD in order to evaluate the optimal thickness of the replacement buffer layer with best TDD/thickness reduction trade-off. A buffer layer sample has been grown based on Fig. 1 with increasing thickness of HT_2 layer and the total thicknesses reaching $500 \mathrm{~nm}$. Fig. 7 shows surfaces of the 270 and 500nm samples observed with AFM and ECCI. The RMS roughness of the 500nm sample is slightly reduced compared to the 270-nm-thick buffer layer, however, a large number of dislocations can be noticed on the surface. These observations have been confirmed by ECCI imaging and TDD count. The dislocation density for the 270-nm-thick buffer layer is relatively high at around $6.1 \pm 0.44$ $\times 10^{8} / \mathrm{cm}^{2}$. Increasing the thickness of the buffer layers to $500 \mathrm{~nm}$ helped to reduce the TDD to around $3.6 \pm 0.33 \times 10^{8} / \mathrm{cm}^{2}$. These results supports the theory that the TDD is proportional to the inverse of the 
film thickness [24]. The most optimal TDD/thickness trade-off is achieved for the thinner buffer layers and the small reduction in TDD of the thicker buffer layers does not justify adding the extra thickness.
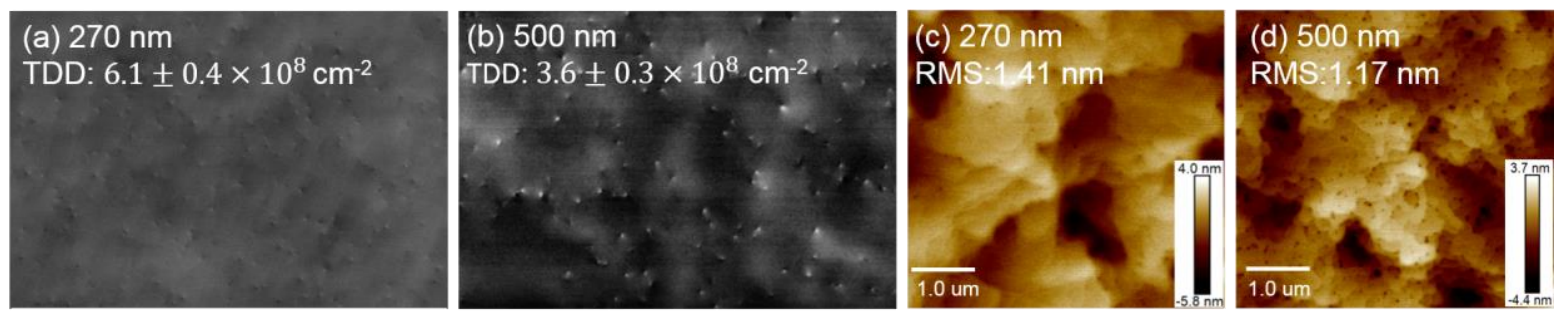

Fig. 7. A comparison of RMS roughness and TDD of 270 and 500nm Ge buffer layers on Si substrates.

\subsection{Effects of doping}

Use of dopants in the growth of buffer layer layers have been shown to reduce the number of threading dislocations and improve crystal quality $[25,26]$. Therefore, the last part of this study investigated the impact of dopants on roughness of the samples and TDD. Another two samples have been grown, which were in situ doped with $5 \times 10^{18} / \mathrm{cm}^{3}$ Baron (B) and Antimony ( $\mathrm{Sb}$ ), respectively, in the low temperature (LT) part of the buffer layer.
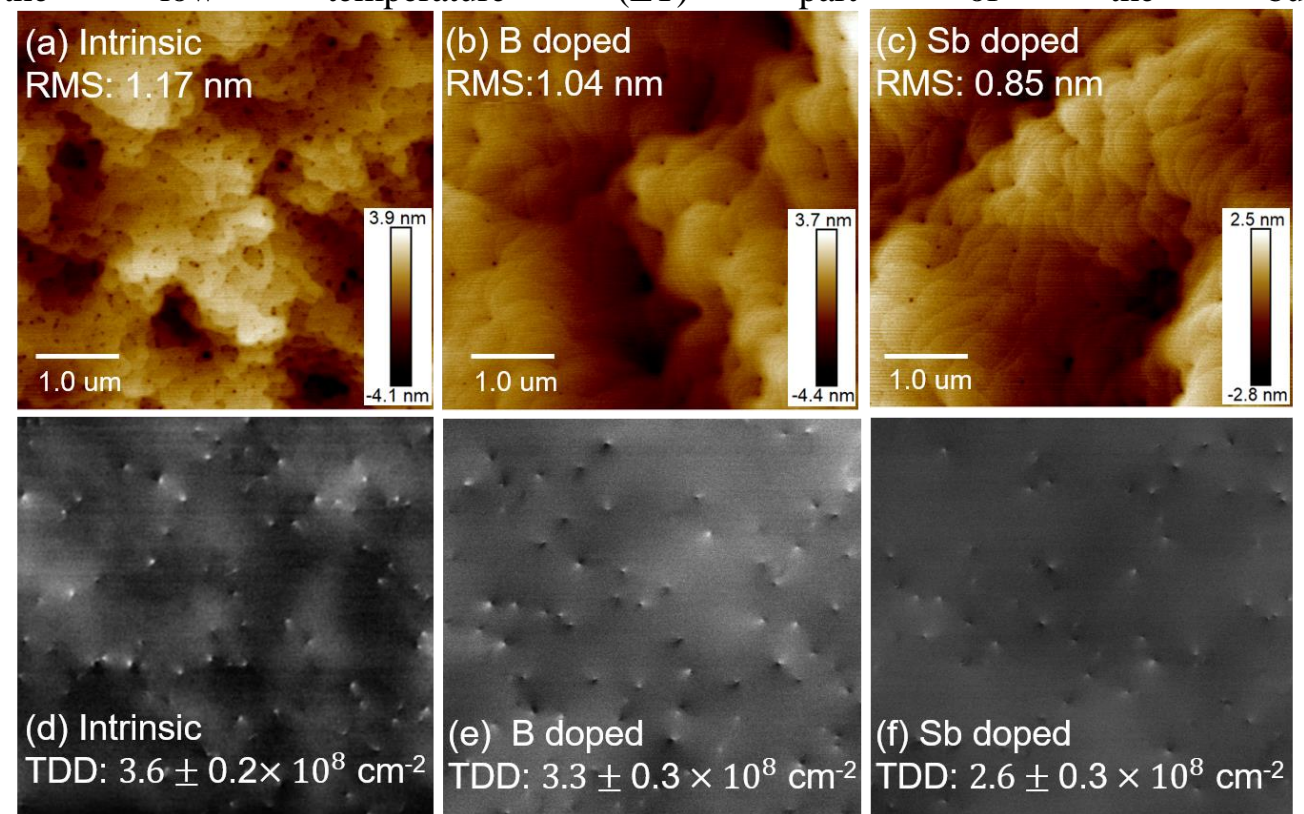

Fig. 8 shows a comparison of surface roughness and threading dislocations between the fully intrinsic, p-type doped (B) and n-type doped ( $\mathrm{Sb}$ ) samples. Comparing the RMS roughness of the three buffer layers (Fig. 8. (a)-(c)), Sb, which acts as surfactant, provide smother surface for the Sb-doped sample than the intrinsic and B doped ones. Also, an obvious reduction of TDD has been observed for Sbdoped one. The implement of Sb enhance the motion of TDs and thus increases the possibility that TDs with opposite Burger vector sign can meet and antihate each other [26, 27]. These results suggest that use of $\mathrm{Sb}$ dopants could improves the crystal quality of the Ge buffer layers. 


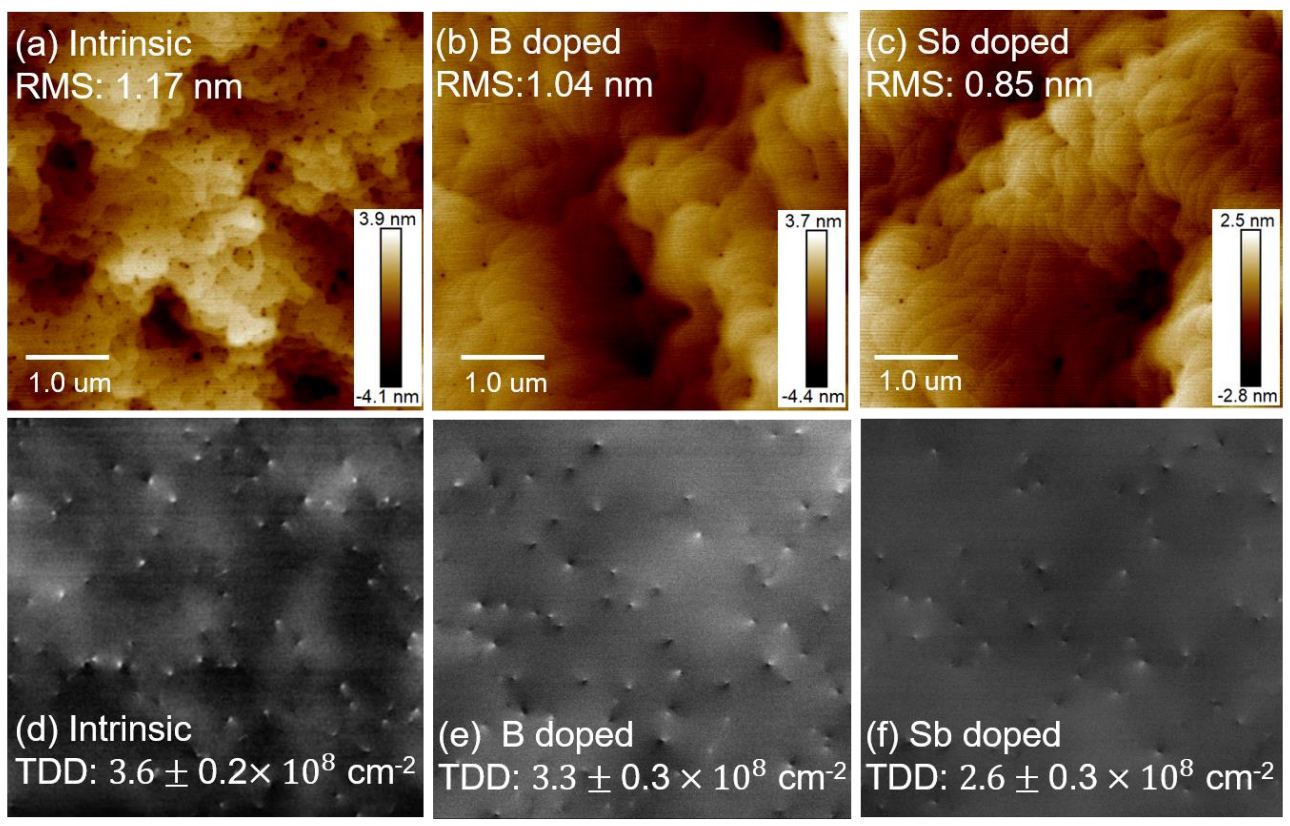

Fig. 8. A comparison of surface roughness and threading dislocation densities of intrinsic, p-doped and n-doped Ge buffer layer samples.

\subsection{InAs/GaAs QD grown on Si substrate with Ge buffer layer layer}

The thin Ge buffer layer is proposed to replace part of the thick GaAs buffer without introducing more defects. The GaAs-based buffer layer consisting of a 1- $\mu$ m-thick bulk GaAs layer followed by three periods of dislocation filter layers was used for the growth of III-V QD laser [7]. When the GaAs buffer layer is approximately $1400 \mathrm{~nm}$ thick, including one dislocation filter layer, the TDD is estimated at around $5.9 \times 10^{8} / \mathrm{cm}^{2}$, which is comparable with the TDD in the 270-nm-thick Ge buffer layer $\left(6.1 \times 10^{8} / \mathrm{cm}^{2}\right.$, Fig. 6.(b)). This means that the total thickness of the buffer layer can be reduced by at least $1 \mu \mathrm{m}$ without changing the TDD of the active layers if that part of the buffer layer is replaced by 200-300 $\mathrm{nm}$ of Ge

In order to confirm high quality of the Ge buffer layer obtained in this work and to demonstrate the possibility of replacing a part of the GaAs buffer layer with a thinner Ge one, a multi-layer selfassembled InAs QD structure was grown. The thin Ge buffer layer used for this sample was 270-nm-thick and annealed at $850^{\circ} \mathrm{C}$ in a $5 \times 5 \mathrm{~min}$ cycle. A Si (100) substrate with a $4^{\circ}$ offcut was used to prevent APBs-related defects. The room-temperature PL of this QD sample is shown in Fig. . The peak wavelength of room temperature PL is at $1297 \mathrm{~nm}$ with full-width at half-maximum (FWHM) equal to $35.7 \mathrm{meV}$. In addition, a $1 \mu \mathrm{m} \times 1 \mu \mathrm{m}$ AFM image of InAs QDs is shown in the top left of Fig. , and a QDs density of about $3.5 \times 10^{10} / \mathrm{cm}^{2}$ is obtained. 


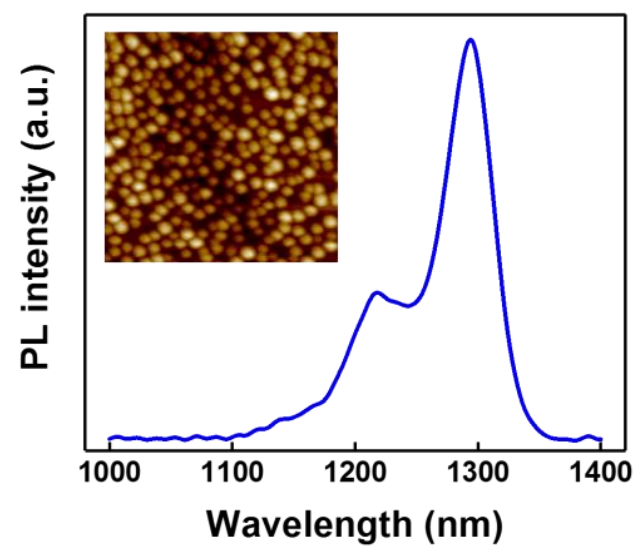

Fig. 9. Room-temperature PL result. inset: $1 \mu \mathrm{m} \times 1 \mu \mathrm{m}$ AFM image of InAs QDs.

\section{Conclusion}

Reducing the total thickness of the buffer layer should help eliminate the wafer cracking problem while keeping the TDD and crystal quality of the III-V active layers at a suitable level. The growth optimisation study described in this paper shown that the TDD density in 270-nm-thick Ge buffer layers on Si can be reduced to $6.2 \times 10^{8} / \mathrm{cm}^{2}$. This has been achieved with an in-situ cyclic annealing method where the buffer layers were heated up to $850^{\circ} \mathrm{C}$ for $5 \mathrm{~min}$ in 5 cycles. Comparison with typical GaAs buffer layer shown that the same TDD can be achieved with $1300 \mathrm{~nm}$ of GaAs. These results confirm that the proposed replacement of a large part of the GaAs buffer layer with a significantly thinner $\mathrm{Ge}$ one is a viable solution. The overall thickness of the samples can be reduced by least $1 \mu \mathrm{m}$ without any negative impact on the quality of the active layers. Moreover, the reduction in thickness can be traded for further reduction of TDD as thinner core buffer layer achieved with Ge allows for additional dislocation filter layers whilst keeping the total thickness within reasonable limits.

\section{Author information}

*Corresponding author: pamela.jurczak@ucl.ac.uk

\section{Acknowledgements}

This work was supported by UK Engineering and Physical Sciences Research Council (EPSRC) with grant number EP/P006973/1 and EPSRC National Epitaxy Facility. J. Yang thanks the China Scholarship Council (CSC) for funding his study.

\section{References}

[1] R. Soref, "The Past, Present, and Future of Silicon Photonics," IEEE Journal of Selected Topics in Quantum Electronics, vol. 12, no. 6, pp. 1678-1687, 2006.

[2] D. A. B. Miller, "Optical interconnects to silicon," IEEE Journal of Selected Topics in Quantum Electronics, vol. 6, no. 6, pp. 1312-1317, 2000.

[3] J. Leuthold, C. Koos, and W. Freude, "Nonlinear silicon photonics," Nature Photonics, vol. 4, no. 8, pp. 535, 2010.

[4] J. Michel, J. Liu, and L. C. Kimerling, "High-performance Ge-on-Si photodetectors," Nature Photonics, vol. 4, no. 8, pp. 527-534, 2010.

[5] G. T. Reed, G. Mashanovich, F. Y. Gardes et al., "Silicon optical modulators," Nature photonics, vol. 4, no. 8, pp. 518, 2010.

[6] D. Liang, and J. E. Bowers, "Recent progress in lasers on silicon," Nature Photonics, vol. 4, no. 8, pp. 511-517, 2010. 
[7] S. Chen, W. Li, J. Wu et al., "Electrically pumped continuous-wave III-V quantum dot lasers on silicon," Nature Photonics, vol. 10, no. 5, pp. 307-311, 2016.

[8] H. Liu, T. Wang, Q. Jiang et al., "Long-wavelength InAs/GaAs quantum-dot laser diode monolithically grown on Ge substrate," Nature Photonics, vol. 5, no. 7, pp. 416, 2011.

[9] E. Tournié, L. Cerutti, J.-B. Rodriguez et al., "Metamorphic III-V semiconductor lasers grown on silicon," MRS Bulletin, vol. 41, no. 03, pp. 218-223, 2016.

[10] M. Kawabe, and T. Ueda, "Self-annihilation of antiphase boundary in GaAs on Si (100) grown by molecular beam epitaxy," Japanese journal of applied physics, vol. 26, no. 6A, pp. L944, 1987.

[11] H. Kroemer, "Polar-on-nonpolar epitaxy," Journal of Crystal Growth, vol. 81, no. 1-4, pp. 193-204, 1987.

[12] S. Mahajan, "Defects in semiconductors and their effects on devices," Acta materialia, vol. 48, no. 1, pp. 137-149, 2000.

[13] M. Tang, S. Chen, J. Wu et al., "1.3- $\mu \mathrm{m}$ InAs/GaAs quantum-dot lasers monolithically grown on Si substrates using InAlAs/GaAs dislocation filter layers," Optics Express, vol. 22, no. 10, pp. 11528-11535, 2014.

[14] I. George, F. Becagli, H. Liu et al., "Dislocation filters in GaAs on Si," Semiconductor Science Technology, vol. 30, no. 11, pp. 114004, 2015.

[15] S. Chen, M. Tang, Q. Jiang et al., "InAs/GaAs quantum-dot superluminescent light-emitting diode monolithically grown on a Si substrate," ACS Photonics, vol. 1, no. 7, pp. 638-642, 2014.

[16] Z. Mi, J. Yang, P. Bhattacharya et al., "High-performance quantum dot lasers and integrated optoelectronics on Si," Proceedings of the IEEE, vol. 97, no. 7, pp. 1239-1249, 2009.

[17] H. Liu, M. Hopkinson, C. Harrison et al., "Optimizing the growth of $1.3 \mu \mathrm{m} \mathrm{InAs} / \mathrm{InGaAs}$ dots-in-a-well structure," Journal of applied physics, vol. 93, no. 5, pp. 2931-2936, 2003.

[18] J. Li, J. Hydrick, J. Park et al., "Monolithic integration of GaAs/InGaAs lasers on virtual Ge substrates via aspect-ratio trapping," Journal of the Electrochemical Society, vol. 156, no. 7, pp. H574-H578, 2009.

[19] X. Huang, Y. Song, T. Masuda et al., "InGaAs/GaAs quantum well lasers grown on exact GaP/Si (001)," Electronics Letters, vol. 50, no. 17, pp. 1226-1227, 2014.

[20] T. Wang, H. Liu, A. Lee et al., "1.3- $\mu \mathrm{m}$ InAs/GaAs quantum-dot lasers monolithically grown on Si substrates," Optics express, vol. 19, no. 12, pp. 11381-11386, 2011.

[21] C. Trager-Cowan, F. Sweeney, P. Trimby et al., "Electron backscatter diffraction and electron channeling contrast imaging of tilt and dislocations in nitride thin films," Physical Review B, vol. 75, no. 8, pp. 085301, 2007.

[22] G. Beltz, M. Chang, M. Eardley et al., "A theoretical model for threading dislocation reduction during selective area growth," Materials Science Engineering: A, vol. 234, pp. 794-797, 1997.

[23] H.-C. Luan, D. R. Lim, K. K. Lee et al., "High-quality Ge epilayers on Si with low threading-dislocation densities," Applied Physics Letters, vol. 75, no. 19, pp. 2909-2911, 1999.

[24] T. Ward, A. M. Sánchez, M. Tang et al., "Design rules for dislocation filters," Journal of Applied Physics, vol. 116, no. 6, pp. 063508, 2014.

[25] M. Gutiérrez, D. Araujo, P. Jurczak et al., "Solid solution strengthening in GaSb/GaAs: A mode to reduce the TD density through Be-doping," Applied Physics Letters, vol. 110, no. 9, pp. 092103, 2017.

[26] K. H. Lee, S. Bao, B. Wang et al., "Reduction of threading dislocation density in $\mathrm{Ge} / \mathrm{Si}$ using a heavily As-doped Ge seed layer," AIP Advances, vol. 6, no. 2, 2016.

[27] J. Patel, and Chaudhuri, "Charged impurity effects on the deformation of dislocation-free germanium," Physical Review B, vol. 143, no. 2, pp. 601, 1966. 